\title{
NOT EVERY DIPOLE IS THE SAME: THE HIDDEN PATTERNS OF DIPOLAR NEAR FIELDS
}

- Michela F. Picardi, Anatoly V. Zayats and Francisco J. Rodríguez-Fortuño

I Physics Department - King's College London - WC2R 2LS, London, UK - DOI: https://doi.org/10.1051/epn/2018402

Nanophotonics is a fast-evolving scientific field studying light at the nanoscale. Its fascinating advances typically stem from concepts in modern physics, such as quantum optics, photonic crystals and optomechanics [1]. Occasionally, new insights appear even from the classical Maxwell's equations of electromagnetism themselves [2]. 
H

ere we outline recently uncovered features of dipoles, the humblest among electromagnetic sources, whose interactions with a nearby structure can nevertheless be unexpectedly rich in terms of their selective excitation of guided modes. This has important applications throughout nanophotonics, from optical information technologies to the design of advanced photonic devices.

Dipolar sources are ubiquitous in every branch of nanophotonics due to their universality. Atoms, quantum dots and other point-like sources, when emitting light, behave as dipoles. Light scattered off any small illuminated particle also does. Matter itself, in its interaction with light, can be regarded as a collection of point dipoles. Dipoles are technologically important; in nanophotonics they are a fundamental tool for the analysis and design of experiments involving near-field instrumentation such as the detector tips in near field optical microscopy or the optical excitations caused by incident electron beams in a cathodoluminescence scanning electron microscope. These kinds of equipment rely on their interaction with optical near-fields of structures - for which the dipole model is perfectly suited. In fact, it is in the properties of these near-field interactions that new behaviours have recently been observed.

\section{Fundamentals of dipolar sources}

Unlike classical gravitational fields, which can be well approximated to first order with knowledge of the total mass of objects, electromagnetic fields may originate from sources with a net zero charge, and so one looks at the next dominant term in the series expansion: the dipole. As we all know, electromagnetic dipoles come in two varieties: electric and magnetic (Fig 1). Electric and magnetic dipole moments $\boldsymbol{p}(t)$ and $\boldsymbol{m}(t)$ vary with time. Fortunately, the superposition principle allows us to consider the monochromatic constituents of any time dependent dipole, such that we may study a harmonically oscillating dipolar source radiating fields of a given, fixed, wavelength. This allows us to eliminate the time-dependence in the dipole moments, at the expense of using complex vectors $\mathbf{p}$ and $\mathbf{m}$ with real and imaginary components accounting for the amplitude and phase of their sinusoidal oscillation. For example, $\mathbf{p}=(1,1,0)$ corresponds to a linearly polarized dipole, oscillating parallel to the $x=y$ line, while $\mathbf{p}=(1, i, 0)$ corresponds to a circularly polarized electric dipole, rotating anticlockwise in the $x y$ plane. The complex nature of the dipole moment, which fully determines its phase, amplitude and polarization, is at the heart of the dipole's remarkable directionality properties. The experimental realization in optical laboratories of electric and magnetic dipoles with determined polarizations is simple. One way is via illumination of small particles which become electrically and magnetically polarized (Fig. 1c). The polarization of the dipolar source that can be generated with this method depends on two factors, the first being the polarization of the fields incident on the particle, which can be easily manipulated. The second factor is the polarizability response of the nanoparticle, which varies in amplitude and phase depending on particle geometry and working wavelength. A fine control on these elements can ensure the possibility to generate any desired dipolar source with an arbitrary polarization.

\section{Directionality of circularly polarized dipoles}

A first hint that not all dipoles are equal came from the stunning, elegant behaviour of circularly polarized dipoles. We always resort to linearly polarized dipoles when we learn, think, and teach about them, with the assumption that any other polarized dipole is just a superposition of linear ones: while true, this neglects the fact that superposition and interference can yield interesting physical effects. Interference is usually associated with the propagation phase acquired by light along different trajectories, but it also applies to the near fields. Fig. 2(a) depicts a linearly polarized dipole $\mathbf{p}=(1,1,0)$ radiating in free space, with its familiar radiation diagram. Fig. 2(b) shows this same dipole placed close to an optical waveguide - and we see that optical modes are excited. This is possible thanks to the dipole nearfields. The coupling interaction between point dipoles
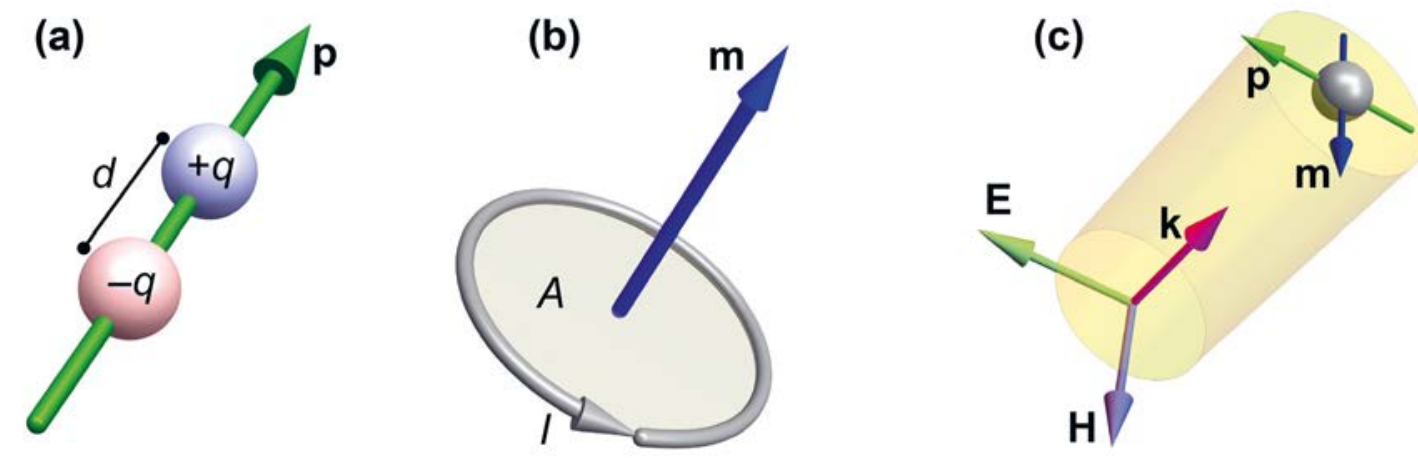

4 FIG. 1: (a) The electric dipole moment vector $\mathrm{p}$ is the product between the charge constituting each of its poles $q$, and their separation distance $d_{\text {. }}$ (b) The magnetic dipole moment vector $m$ is the product between the amplitude of a circulating current I and the area it encloses Â̂, in a direction î normal to the area. Both converge to point sources in the appropriate limit. (c) A polarizable particle being illuminated by an incident beam with wave-vector k, electric field E, and magnetic field H. The particle becomes polarized and behaves like a dipolar source with dipole moments proportional to the incident fields $\mathrm{p}=a_{e} \mathrm{E}$ and $m=a_{m} \mathbf{H}$ with $a_{e / m}$ representing complex proportionality constants, which are the particle's electric and magnetic polarizability. 


\section{BOX 1. A VARIETY OF EXPLANATIONS FOR THE NEAR-FIELD DIRECTIONALITY OF DIPOLES CAN BE GIVEN, ALL BEING ULTIMATELY EQUIVALENT, BUT EACH HIGHLIGHTING DIFFERENT ASPECTS.}

\section{- Phase matching between the dipole fields and the waveguide:} the phase matching of fields along smooth interfaces requires that the moving wave-fronts of a source must match the moving wavefronts of the mode being excited. Consider the fields radiated from the circular dipole depicted in Fig. 2(c). Its wave-fronts are counterclockwise rotating spirals. Clearly, if a waveguide is placed below the dipole as in Fig. 2(d), the wave-fronts sweep the waveguide from left to right, matching the wave-fronts of the guided mode propagating in the waveguide in the left to right direction, which gets excited. Indeed, placing the waveguide above the dipole would switch the directionality to the left, in agreement with a waterwheel analogy.

\section{Superposition of excitations with different symmetries: consider a} vertically polarized dipole $\mathbf{p}=a \hat{\mathbf{y}}$, over a dielectric waveguide. Due to the complete mirror symmetry of the problem, modes are excited in the waveguide in both directions, exactly in phase. The field will have an even symmetry with respect to the mirror-symmetry plane. Now consider a horizontal dipole $\mathbf{p}=b \hat{\mathbf{x}}$. This dipole is oscillating, alternatively pointing left and right, and this will evidently excite modes in both directions with identical amplitude, but exactly out of phase, constituting an odd symmetry of excitation. By a judicious superposition of both, horizontal and vertical components of the dipole result in the addition of an even and an odd function, which may produce a highly asymmetrical total field resulting in unidirectional excitation. This is the case for the circular dipole. In general, any dipole $\mathbf{p}$ and $\mathbf{m}$ is a superposition of six components, which can all interfere between each other.

\section{Overlap of the dipole's fields with the guided fields: the amount} of coupling between a dipolar source $\mathbf{p}$ and $\mathbf{m}$, and a waveguided mode with electric and magnetic fields $\mathbf{E}$ and $\mathbf{B}$ (evaluated at the location of the dipole) is given by Fermi's golden rule $\left|\mathbf{p}^{*} \cdot \mathbf{E}+\mathbf{m}^{*} \cdot \mathbf{B}\right|^{2}$ involving dot products between dipole moments and fields, where * signifies complex conjugation [11]. In the case of the circularly polarized electric dipole, the excitation along one direction is zero because $\mathbf{p}^{*} \cdot \mathbf{E}=0$ for the field of the mode in that direction. For example, $(1, i, 0)^{*} \cdot(1,-i, 0)=0$. This happens because the electric field of the waveguided mode indeed has a near circular polarization $\approx(1$, $\pm i, 0$ ) whose handedness depends on its propagation direction, a phenomenon named spinmomentum locking [12], [13]. In a more general case, the expression $\mathbf{p}^{*} \cdot \mathbf{E}+\mathbf{m}^{*} \cdot \mathbf{B}$ is composed of the sum of six different terms, corresponding to how each component of the dipole excites each mode: this sum may easily interfere destructively for certain dipoles and waveguide modes [14]. This explanation gives an equal focus to both the dipoles and the fields of the specific waveguide, betraying the fact that the directionality is a universal property of the dipole, valid for any waveguide. It has the advantage of being an exact valid explanation in any scenario, such as dipolar sources inside photonic crystals.

- Angular spectrum of the dipolar fields: This explanation mathematically fleshes-out the simple phase matching argument given above. It is most powerful when the dipole is placed near smooth interfaces which conserve momentum and thus allow us to apply phase matching arguments. With it, we realize that the directionality of the dipole is a property of the dipole itself, universal for any waveguide [15]. It is based on a careful analysis of the fields produced by a dipole [16], whose exact analytical form has been known for as long as Maxwell's equations themselves. The amplitude and phase of the electric field $\mathbf{E}(\mathbf{r})$ originating from a dipole, at any point in space $\mathbf{r}$, can be written in a short single-line equation. We may then analyse this field by means of its spatial Fourier transform $\mathbf{E}(\mathbf{r})=\iiint d \mathbf{k} \mathbf{E}(\mathbf{k}) e^{i \mathbf{k} \cdot \mathbf{r}}$ where $\mathbf{E}(\mathbf{k})$ is the constant electric field amplitude of a plane wave or evanescent wave with wave-vector $\mathbf{k}=\left(k_{x}, k_{y}, k_{z}\right)$ and $e^{i \mathbf{k} \cdot \mathbf{r}}$ describes its propagation or attenuation through space. We have "spread out" the field into its spatial components, called its spatial or angular spectrum. In the phase matching argument above, we pointed out from an intuitive and hand-wavy argument that the phase fronts of a circularly polarized dipole sweep from left to right when looking below the dipole, explaining its directionality. Indeed, the angular spectrum of such dipole reveals the dominance of evanescent components with wave-vectors $\mathbf{k}$ pointing from left to right when looking from below the dipole [15], providing a solid quantitative foundation to our intuitive notion. Janus and Huygens dipoles exhibit similar imbalances in their angular spectra, fully explaining their behaviour and symmetries. 
and nearby waveguides is a well-known property of dipolar sources. Fig. 2(c) shows a circularly polarized dipole $\mathbf{p}=(1, i, 0)$ radiating in free space, and Fig. $2(\mathrm{~d})$ shows the same dipole near an optical waveguide [3]. We see that, surprisingly and despite the mirror symmetry of this structure, the circularly polarized dipole excites modes in a single direction of the waveguide. This is a fundamental behaviour of the dipole, inherently broadband, robust to losses and imperfections. Remarkably, it admits many different but equivalent explanations, detailed in Box 1. The excitation of light modes from this circularly polarized dipole resembles a water wheel for light. When a circularly polarized dipole is sandwiched between two waveguides (Fig 3a), both are excited in opposite directions. Known for a few years now, this unique directionality has yielded fascinating applications such as ultracompact broadband optical nanorouting [3], [4], polarization analysers [5], quantum optical applications [6] including non-reciprocal nanophotonic devices [7], exotic lateral optical and Casimir forces [8], [9], generation of polarized light [10], and many others. And yet this dipole is but a first taste of how not every dipole is the same.

\section{Beyond circularly polarized dipoles: a zoo of directional dipoles}

Using the analysis techniques described in Box 1, we recently asked ourselves what other unconventional dipolar sources could be found. By combining electric and magnetic dipoles together, new directional dipolar source behaviours are revealed [14]. The idea of combining electric and magnetic dipoles to obtain distinct functionality is not new. The Huygens dipole is a source which combines two linearly polarized orthogonal electric $\mathbf{p}$ and magnetic $\mathbf{m}$ dipoles with an amplitude ratio of $p=m / c$, where $c$ is the speed of light, such that their combined radiation is directional in the far-field in the $\mathbf{p} \times \mathbf{m}$ direction. For example, $\mathbf{p}=(1,0,0)$ and $\mathbf{m}=(0, c, 0)$ show complete far field directionality in the $+z$ direction. The radiation of the electric and magnetic dipoles, omnidirectional when on their own, interfere destructively in one direction but constructively in the opposite
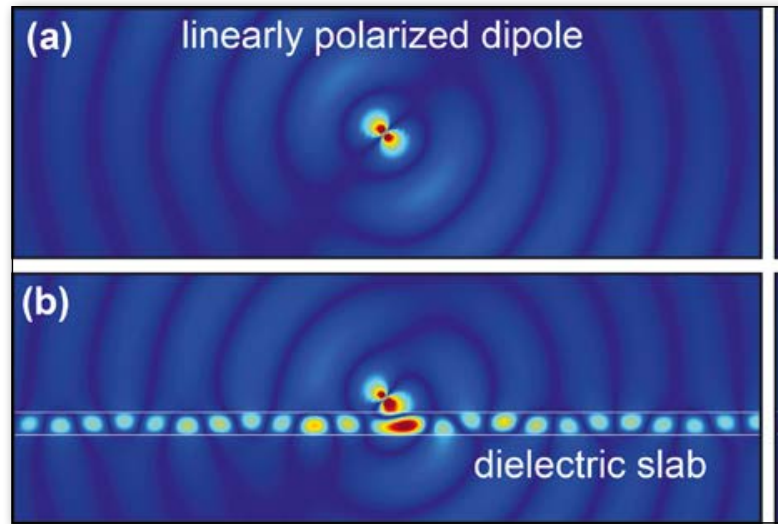

one, resulting in unidirectionality. This has been used to create engineered surfaces with perfect reflection, and all-dielectric mirrors among other applications [17]. It turns out that the same phenomenon exists in the dipole's near-field [14]. The Huygens dipole exhibits a different symmetry of near-field excitation compared to the circular one: when sandwiched between waveguides, it couples to the modes in the waveguides along the same direction for both (Fig. 3b).

Both circular and Huygens' dipole appear as solutions of a single equation, yet a third solution completes the set [14]. This novel dipole greatly resembles the Huygens' dipole, in that it too is a combination of linearly polarized and orthogonal dipoles, but its electric and magnetic dipoles are in quadrature phase, that is, with $a / 2$ phase difference between dipole moment amplitudes. It does not show any directional behaviour in the far-field, and yet it shows unique and surprising properties in its near field coupling. This source, like its namesake ancient Roman god Janus, has two faces. One face enables coupling into nearby waveguides, while the opposite face is non-coupling to them. Therefore, flipping the Janus dipole's face as if it were a coin, enables switching the coupling on or off. Equivalently, the binary state of the coupling depends on which side of the dipole the waveguide is placed. When sandwiched between two waveguides, the Janus dipole couples to both directions on the same waveguide, the one facing its coupling side, while blatantly ignoring the other one (Fig. 3c). These new near-field dipolar sources all follow the same explanations given in Box 1, ultimately relying on near-field interference between distinct dipolar components.

\section{Conclusions}

The three elemental dipoles shown in Fig. 3 arise as solutions from one same equation, describing dipole directionality in a simplified planar waveguide scenario [14]. In fact, any linear combination of the three elemental sources results in an infinite range of possible directional dipole sources. More complex three-dimensional scenarios and more complex waveguide geometries will enable even further examples of directional

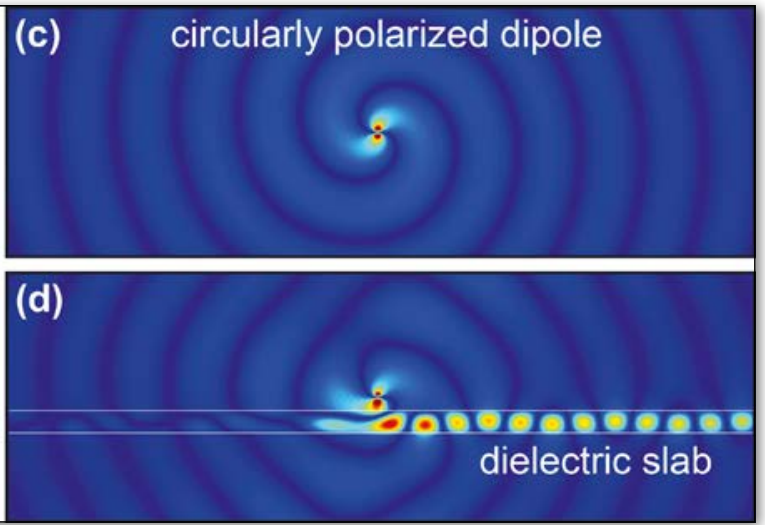

4 FIG. 2:

Snapshot of radiated magnetic field amplitude of a linearly $(a, b)$ and circularly $(\mathrm{c}, \mathrm{d})$ polarized electric dipole radiating either in freespace $(a, c)$ or at a subwavelength distance $0.1 \lambda$ near a dielectric waveguide $(b, d)$. The phenomenon exists for any kind of waveguide. In this figure we chose a dielectric slab with refractive index $n=3$ and thickness $t=0.2 \lambda$. 
-FIG. 3: Behaviour of three different dipolar sources: (a) circular (b) Huygens and (c) Janus dipoles, sandwiched between two standard silicon

nanophotonic

waveguides. The

dipole moments

where optimized for optimal directionality contrast as described

in Ref. [14]. The

figure renders a

snapshot of the field

intensity of a real

three dimensional

numerical simulation.

The simulation

was performed in

the commercial

electromagnetic

solver CST Microwave

Studio. The fields

are shown shifted in

space so that they

appear above the

waveguides instead

of inside. The cross

section of these

silicon waveguides is $(0.2 \times 0.16) \lambda$. The refractive index of silicon at $\lambda=1550 \mathrm{~nm}$ was taken as $n=3.45$. Notice that the three sources are intimately related to the three spatial symmetries

of the underlying structure: two mirror symmetries along orthogonal planes, and a half-turn rotational symmetry. dipoles, opening a zoo of possibilities. The behaviour is fundamental and robust. Given the amount of applications that arose from circularly polarized dipoles alone, we expect these new dipolar possibilities to give rise to a plethora of new ideas. As a simple example, the directionality of circularly polarized dipoles was used as a method for local measurement of the transverse electric field polarization: the generalization of the concept will allow local measurement of all spatial components of both electric and magnetic fields in complex structured electromagnetic beams. New ideas can arise in a wide array of areas such as quantum optics, optical logical circuits, photonic nanorouting, polarization detection and synthesis, novel optical forces and torques in nanoparticles, and hopefully in as yet unforeseen devices throughout nanophotonics and other regions of the electromagnetic spectrum.

\section{Acknowledgements}

This work was supported by European Research Council Starting Grant ERC-2016-STG-714151-PSINFONI and EPSRC (UK).A.Z. acknowledges support from the Royal Society and the Wolfson Foundation. Authors thank Dr. Paolo Biagioni for his title-inspiring tweet https://twitter. com/pbiagio78/statuses/974359896492838912.

\section{About the authors}

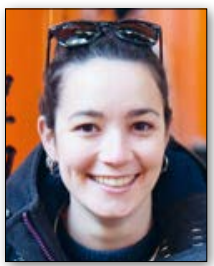

Michela. F. Picardi is a $\mathrm{PhD}$ student at King's College London, UK, under the supervision of Francisco J. Rodríguez-Fortuño. Her project is devoted to the exploration of degrees of freedom of light and their mutual interactions, as well as their behaviour in nanophotonic and plasmonic environments for future lightbased technologies.

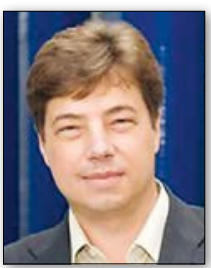

Anatoly V. Zayats is a Professor of Experimental Physics and the head of the Photonics and Nanotechnology Group at the Department of Physics, King's College London. His current research interests include among others nanophotonics, plasmonics, metamaterials, and lowdimensional structures. He is a Fellow of the Institute of Physics, the Optical Society of America, SPIE and the Royal Society of Chemistry.

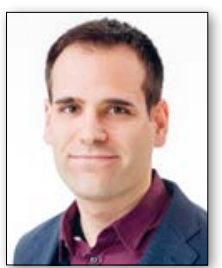

Francisco J. Rodríguez-Fortuño is a Lecturer at the Department of Physics, King's College London. His research focuses on plasmonic devices, optical forces, optical nanoantennas, metamaterials and novel electromagnetic phenomena. Francisco is a member of the editorial board of Scientific Reports and is a principal investigator on the European Research Council Starting Grant 714151 PSINFONI.

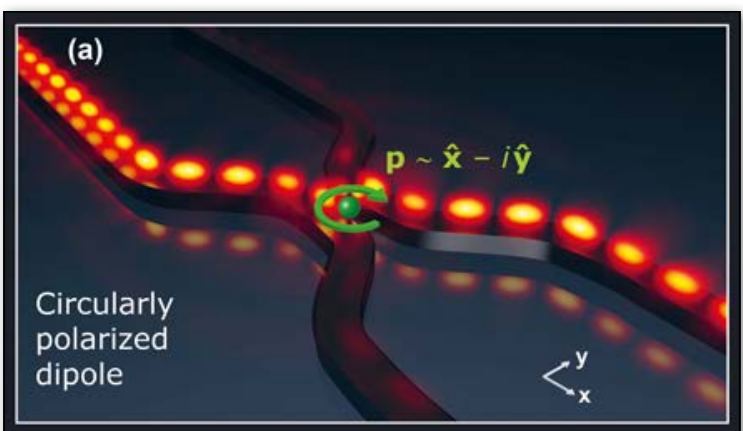

(b)
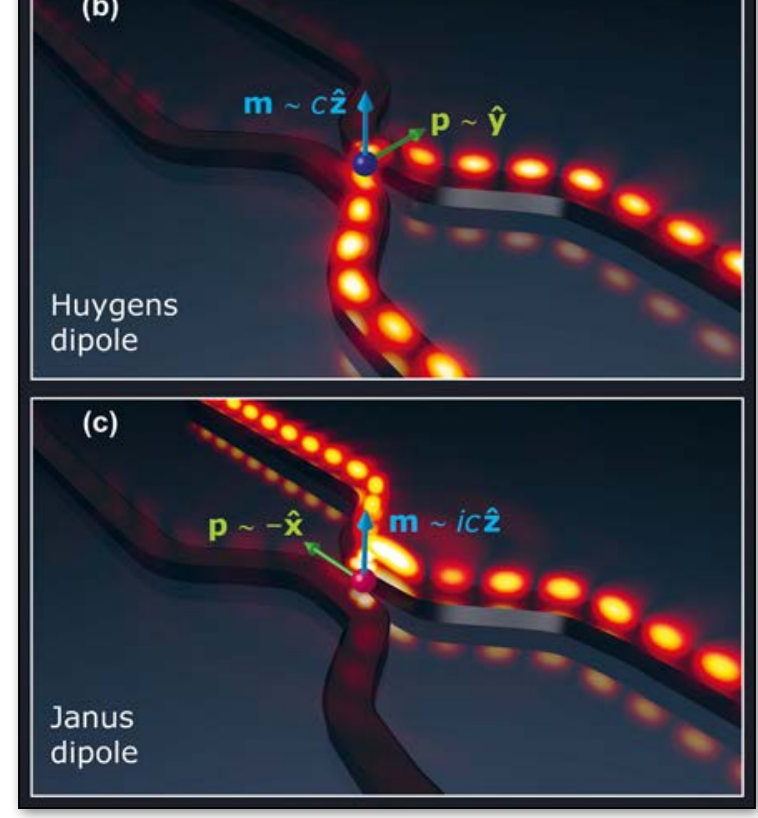

\section{References}

[1] J. Heber and A. Trabesinger, Nature milestones: photons. Nat. Mater. 9, S5-S20 (2010)

[2] N. Engheta, Science 349-6244, 136 (2015).

[3] F. J. Rodriguez-Fortuño et al., Science 340-6130, 328 (2013).

[4] J. Petersen, J. Volz, and A. Rauschenbeutel, Science 346-6205, 67 (2014).

[5] A. Espinosa-Soria, F. J. Rodríguez-Fortuño, A. Griol, and A. Martínez, Nano Lett. 17-5, 3139 (2017).

[6] L. Marrucci, Nat. Phys. 11-1, 9 (2014).

[7] M. Scheucher, A. Hilico, E. Will, J. Volz, and A. Rauschenbeutel, Science 354- 6319, 1577 (2016).

[8] F. J. Rodríguez-Fortuño, N. Engheta, A. Martínez, and A. V. Zayats, Nat. Commun. 6, 8799 (2015).

[9] A. Manjavacas, F. J. Rodríguez-Fortuño, F. J. García de Abajo, and A. V. Zayats, Phys. Rev. Lett. 118-13, 133605 (2017)

[10] F. J. Rodríguez-Fortuño, D. Puerto, A. Griol, L. Bellieres, J. Martí, and A. Martínez, Laser Photon. Rev. 8-3, L27 (2014).

[11] B. le Feber, N. Rotenberg, and L. Kuipers, Nat. Commun. 6, 6695 (2015)

[12] A. Aiello, P. Banzer, M. Neugebauer, and G. Leuchs, Nat. Photonics 9-12, 789 (2015).

[13] K. Y. Bliokh, D. Smirnova, and F. Nori, Science 348-6242, 1448 (2015)

[14] M. F. Picardi, A. V. Zayats, and F. J. Rodríguez-Fortuño, Phys. Rev. Lett. 120-11, 117402 (2018).

[15] M. F. Picardi, A. Manjavacas, A. V. Zayats, and F. J. Rodríguez-Fortuño, Phys. Rev. B 95-24, 245416 (2017).

[16] J. D. Jackson, Classical Electrodynamics. Wiley, 1998.

[17] J. M. Geffrin et al., Nat. Commun. 3-1, 1171 (2012). 